\title{
¿SIEMPRE ESTRUCTURAS Y VOCABULARIO?
}

\author{
Marina Kirac (Universidad Nacional de Lanús - \\ Instituto Nacional Superior del Profesorado Técnico \\ de la Universidad Tecnológica Nacional)* \\ marinakirac@gmail.com \\ Martha Cecilia Ortigueira (Instituto Nacional Superior del Profesorado \\ Técnico de la Universidad Tecnológica Nacional)** \\ marthaortigueira@usa.net \\ Omar Alberto Villarreal (Instituto Nacional Superior del Profesorado \\ Técnico de la Universidad Tecnológica Nacional) ${ }^{* * *}$ \\ omarvillarreal@speedy.com.ar
}

Recibido: 2/07/2012 Aceptado: 18/11/2012

\section{Resumen}

Los métodos de enseñanza de la lengua extranjera han cambiado de manera significativa en el transcurso del tiempo. Sin embargo, el contenido y la forma en que se presenta la lengua a los alumnos sigue, en la amplia mayoría de los casos, sin experimentar grandes cambios. La tendencia de enseñar gramática y vocabulario (lexis) separadamente es moneda corriente en la práctica de los docentes de inglés como lengua extranjera. De esta manera el fin principal de la tarea que es enseñar la lengua como herramienta de comunicación y expresión parece dejarse de lado. El propósito del siguiente trabajo es reflexionar sobre nuestro pensamiento y convic-

* Magister en la Enseñanza del Inglés como Lengua Extranjera. Universidad de Jaén. Licenciada en Gestión Educativa. Universidad Nacional de Lanús. Profesora en Inglés e Inglés Técnico. Instituto Nacional Superior del Profesorado Técnico. Universidad Tecnológica Nacional.

** Doctora en Lenguas Modernas. Universidad del Salvador. Profesora en Inglés y Profesora en Enseñanza Elemental de Inglés. Facultad de Filosofía y Letras. Universidad Católica Argentina.

*** Doctor en Lenguas Modernas. Universidad del Salvador. Licenciado en Ciencias de la Educación. Universidad Católica de La Plata. Profesor en Inglés e Inglés Técnico. Universidad Tecnológica Nacional. 
ciones como profesores de inglés sobre la lengua y su enseñanza y la importancia de "darnos cuenta" (en inglés noticing) de forma tal que podamos llevar la teoría a la práctica dentro del ámbito áulico. Al enseñar desde este punto de vista, lograremos que la gramática y el vocabulario interactúen. Desarrollaremos esta posición desde el enfoque del Método Léxico (Lexical Approach), sugiriendo estrategias para desarrollar el aprendizaje y enseñanza de la lengua desde una perspectiva diferente que sirva para desarrollar las habilidades comunicativas de los alumnos teniendo en cuenta sus diversos potenciales haciendo referencia a la teoría de la inteligencias múltiples.

\title{
Palabras Clave
}

Método léxico - Noticing - Lengua extranjera - Estrategias - Habilidades comunicativas.

\begin{abstract}
Foreign language teaching methodologies have significantly changed over the past few years. However, the content and the way in which the target language is presented to learners have not undergone apparently further changes. For most teachers of English as a Foreign Language, the idea of teaching and learning languages meant basically to divide their task into two separate practices: teaching grammar and teaching vocabulary, learning structures and learning words. In this view the main purpose of teaching the language as a means of communication was not thoroughly considered. The purpose of the following paper is to reflect upon our thoughts and beliefs about the language and its teaching and the importance of noticing so that theory can be put into practice. The before stated ideas will be expanded under the scope of the Lexical Approach and some strategies based on the Theory of Multiple Intelligences will be suggested in order to help teachers develop teaching and learning skills that aim to foster and enhance students' communicative skills.
\end{abstract}

\section{Key words} skills.

Lexical approach - Noticing - Foreign language - Strategies - Communicative

\section{Introducción}

¿Qué enseñamos cuando enseñamos? ¿Qué hay detrás de nuestra planificación, plan de clase? ¿Cuánto de nosotros mismos va con nuestra enseñanza? ¿Cuán comprometidos estamos con nuestra práctica docente? ¿Somos realmente conscientes de la influencia abrumadora a la que nuestros alumnos están expuestos cuando nos escuchan? ¿Notan estos alumnos cómo ponemos nuestras ideas en acción o se limitan a observar en silencio?

Las palabras que usamos son nuestra forma de expresarnos y transmitir nuestras ideas, materializar nuestros pensamientos, expresar sentimientos, evocar las imágenes que recorren la mente, participar e interactuar dentro del medio en que vivimos. Nuestras palabras allanan el camino para la comunicación. 
Cabe preguntarse entonces cuán efectiva será esta comunicación y si realmente consideramos la lengua como vehículo de comunicación y trasladamos este concepto a nuestra tarea docente y por ende a nuestros alumnos. Es válido entonces cuestionarse cuánto de nosotros va implícito en lo que decimos y si realmente nuestras palabras comunican eficientemente lo que queremos decir.

A través de los años los métodos de enseñanza y aprendizaje de la lengua, y en el caso particular del inglés como lengua extranjera han experimentado infinidad de cambios. En los últimos treinta años un importante número de propuestas sobre cómo enseñar de manera eficiente y productiva una lengua extranjera han sido estudiadas, puestas en práctica y adoptadas por los docentes del área. Sin embargo, el contenido y en la mayoría de los casos la forma de presentar la otra lengua no han sido sujeto de tanta modificación. En otras palabras, es casi posible sugerir que los presuntos cambios metodológicos y de enfoque en cuanto a la enseñanza del inglés como lengua extranjera, no han sido tales. Por ejemplo, hay una fuerte y arraigada tendencia dentro de los profesores de inglés de enseñar vocabulario y gramática separadamente, como dos asignaturas existencialmente diferentes y hasta antagónicas. Para muchos, la idea de aprender y enseñar un idioma significaba y aun significa básicamente dividir la tarea en dos prácticas separadas e independientes: enseñar gramática y enseñar vocabulario, aprender estructuras y aprender palabras, evaluar conocimientos de reglas y conocimientos de vocabulario. La lengua parece entonces constituir dos mundos separados e independientes que no se interrelacionan: el mundo de las estructuras y el mundo del vocabulario. Cabe entonces preguntarse si puede una misma lengua reflejarse en un espejo con dos caras distintas y hasta percibidas como diferentes.

Para enseñar inglés como lengua extranjera desde un enfoque "léxico" debemos dejar de considerar la gramática y el vocabulario en forma independiente. Adoptar un enfoque léxico de la enseñanza de la lengua extranjera significa concebir la lengua como Lexis: palabras y combinaciones, frases y grupos de palabras que usamos como unidades para comunicar, expresar sentimientos, representar ideas, transmitir información y tantas otras funciones del lenguaje. Estas unidades se denominan en inglés "chunks" (trozos). La lengua por ende se compone de "chunks" que se pueden combinar y de esa forma dar origen a textos (nos referimos aquí al decir texto a producciones tanto orales como escritas) continuos y coherentes. Usamos la palabra continuos para referirnos a un proceso que sucede sin interrupción y coherente porque está bien organizado y es fácil de entender.

A pesar de su popularidad, sin embargo, poco se ha hecho hasta el momento para implementar el método léxico tanto en diseños curriculares como en libros de texto. Por cierto, es muy poca la opción de libros de texto para aquellos profesores que desean implementar esta forma de enseñanza en sus aulas. 
Un motivo de preocupación que a la vez complementa la idea de optimizar la tarea de enseñanza de la segunda lengua expuesta en el párrafo anterior es encontrar la manera de captar la atención y el interés de nuestros alumnos, tanto niños como adolescentes y adultos de manera de hacer de nuestra tarea algo fructífero. Cotidianamente nos cuestionamos si la manera de presentar el material en cuestión es el adecuado, si los resultados obtenidos son los esperados o si bien podrían haber sido superados, si los alumnos aprenden con facilidad, o qué es lo que dificulta el aprendizaje. Básicamente la participación y el interés de nuestros discípulos deben ser motivos constantes de atención. Para llegar a los alumnos en forma efectiva y eficiente deberemos tener en cuenta algo más que el programa de estudios o el material a utilizar, debemos tener en cuenta a nuestros alumnos como individuos partícipes indispensables del proceso de enseñanza y aprendizaje.

Es importante que como profesionales dedicados a la enseñanza seamos conscientes de las diferentes capacidades y destrezas de los individuos que aprenden, así como de sus distintos estilos de aprendizaje y tratemos de atender sus necesidades. Una valiosa herramienta de la cual podemos valernos a fin de lograr dicho objetivo es la Teoría de las Inteligencias Múltiples desarrollada por el Dr. Howard Gardner (1983). Esta teoría que representa un desafío al concepto tradicional de inteligencia ha sido recibida con entusiasmo dentro del ámbito de la educación a nivel mundial constituyendo un recurso valioso para facilitar la tarea de ambas partes involucradas dentro del proceso de enseñanzaaprendizaje. Es cierto que la idea de presentar el material de clase tratando de favorecer el desarrollo de cada uno de los ocho potenciales o inteligencias que cada individuo posee es prácticamente imposible de efectivizar y poner en práctica en el aula en forma diaria. Sin embargo, si este modelo de trabajo sirve para acrecentar una o dos inteligencias que de otra manera no hubiesen sido explotadas entonces ha servido su propósito realmente.

El propósito del siguiente trabajo será por lo tanto examinar los principios centrales del método léxico y hacer una breve reseña de propuestas didácticas para su uso en clases de inglés como lengua extranjera. Propondremos, además, una serie de actividades de clase para aplicar la teoría de la inteligencias múltiples que pueden usarse tanto en la clase de idioma extranjero como en cualquier otra clase.

\section{Método Léxico}

El Método Léxico (Lexical Approach), creado por Michael Lewis se concentra en desarrollar las competencias y destrezas de los alumnos con el léxico, o palabras y las diferentes combinaciones de palabras. Este método se basa en que una parte muy importante de la adquisición de la lengua para comprender y producir frases como un "todo no analizado", o unidades léxicas (en inglés chunks) y en que dichos chunks se convierten en la "información prima" por 
la cual percibimos modelos de lenguaje tradicionalmente pensados como gramática (Lewis, 1998, p. 95). La enseñanza de la lengua, entonces se focaliza, según Lewis, en expresiones fijas que ocurren con alta frecuencia, por ejemplo "Lo siento", "Eso no me va a pasar a mí", "Gracias por todo", "Por supuesto", más que en oraciones originalmente creadas (1998).

Michael Lewis sugiere lo siguiente:

- Las palabras (lexis) son la base de la lengua.

- Las palabras se malinterpretan al enseñar la lengua porque se presume que la gramática es la base del lenguaje y que dominar el sistema gramatical es un pre requisito para la comunicación efectiva.

- El principio clave de este método es que "la lengua consiste en léxico gramaticalizado, y no gramática lexicalizada".

- Uno de los principios centrales de organización de cualquier programa de enseñanza centrado en el significado serán las palabras (Lexis)

En el Método Léxico, el vocabulario en sus distintas formas y expresiones tiene un rol central en la enseñanza y aprendizaje de la lengua. Dentro de las actividades utilizadas para desarrollar el conocimiento del léxico de los alumnos es posible nombrar entre otras:

- Lectura y comprensión auditiva (intensiva y extensiva) en la lengua que se está aprendiendo.

- Comparación y traducción entre la lengua materna y la extranjera- no palabra por palabra sino por partes (chunks).

- Imaginar los significados de los elementos del vocabulario según el contexto.

Por ende, para enseñar la lengua desde este enfoque es necesario:

1) Tener el conocimiento necesario de:

- La lengua que queremos enseñar

- Cómo enseñar esta lengua

- Cómo aprenden los alumnos

- Cómo aprendemos nosotros mismos

- Habilidades interpersonales

2) Explotar al máximo el tiempo disponible en clase para desarrollar estrategias que ayuden a los alumnos a

- Manejar ítems no conocidos.

- Registrar y recordar el nuevo lenguaje de forma eficiente.

- Aumentar la conciencia de la lengua que los alumnos tienen a través de actividades de tipo receptivo.

- Utilizar el lenguaje aprendido

- En las primeras etapas del proceso de aprendizaje, los alumnos se encontrarán con más lenguaje del que son ellos mismos capaces de 
producir. Esto es similar en cierta forma al proceso de adquisición de la lengua materna, en el que el niño está expuesto a la lengua en cuestión en cantidades mayores a las que será capaz de producir por si mismo. Según Lewis (1993) muchos de los principios para la adquisición de la lengua materna son de crucial importancia para la adquisición de la segunda lengua o en nuestro caso en particular el de la lengua extranjera. Dentro de dichos principios destacaremos,

- Una lengua nos se aprende aprendiendo sonidos por separado y estructuras y luego combinándolos.

- La gramática se adquiere a través de un proceso de observación, hipótesis y experimentación.

- Es posible utilizar frases completas sin entender el significado en particular de cada una de sus partes. (Islam, 2003)

Para algunos profesores utilizar este tipo de metodología de enseñanza implicará cambios sustanciales en el método de trabajo utilizado en su práctica diaria. Los cambios constituyen un desafío constante que debemos afrontar, es enfrentarnos a algo distinto y tomar decisiones, es estar abiertos a nuevas experiencias, en definitiva es crecer. Enseñar es encontrar y aceptar un desafío real. Los alumnos tienen que aprender, también, a asumir el desafío que significa aprender una nueva lengua e involucrarse en el proceso y tomar conciencia de la importancia que ellos tienen como elemento indispensable de su propio aprendizaje. El aprendizaje es un proceso voluntario. Aprendemos cuando queremos aprender.

\section{Unidades léxicas}

Aprender los componentes de una lengua y el vocabulario en particular constituyen una tarea ardua. Muchas veces los alumnos no son conscientes de la importancia que tiene el aprendizaje del vocabulario ya que tradicionalmente la idea de poder aprender un idioma satisfactoriamente se asocia a saber su gramática y muchas horas y esfuerzo se dedican a tal fin. Sin embargo, no es siempre un error gramatical el que va a impedir la comunicación. Según Higueras (2009), refiriéndose a los alumnos,

... si tenemos en cuenta, por una parte, que en el mundo real en el que van a utilizar esa lengua suelen ser los errores léxicos los que dificultan la comprensión y que los errores gramaticales se toleran mucho mejor que los léxicos y, por otra, que el léxico no será un fin en si mismo, sino un medio para comunicar y mejorar la competencia comunicativa, estaremos entonces de acuerdo en que el profesor tiene que resaltar la necesidad de aprender léxico desde los primeros días del aprendizaje de una lengua. 
Michael Lewis (1998) sugiere utilizar el concepto más amplio de "unidades léxicas" (chunks) en lugar de "palabra" y distingue básicamente dos tipos:

1. Palabra, refiriéndose a la unidad en si, como por ejemplo: agua, felicidad, escribir, azul, estudioso.

2. Varias palabras, como por ejemplo: había una vez, café amargo, sin embargo, mucho gusto, morirse de risa.

A la vez estas unidades léxicas pueden dividirse en tres tipos (Higueras, 2009):

a) Las frases hechas. Son expresiones fijas de la lengua como por ejemplo: meter la pata, patear el tablero, medir las consecuencias, salir de compras, tomar asiento. El significado de estas frases se interpreta en conjunto, su significado no refleja la suma de los significados de las palabras que la forman.

b) Las combinaciones sintagmáticas (en inglés collocations). Sin ser frases hechas son palabras que tienden a aparecer juntas. Es de suma importancia no solo enseñar distintas combinaciones sino también hacer que los alumnos las descubran (Una manera de darse cuenta del uso de estas combinaciones es hacerlo también con la lengua materna y pedir a los alumnos que observen cuáles pueden notar).

Para entender el concepto de combinación sintagmática y su importancia para la enseñanza y aprendizaje del léxico, podemos señalar que en la lengua hay restricciones que impiden que palabras del mismo significado aparezcan en los mismos contextos. Por ejemplo, mientras la palabra marrón y moreno/a se refieren al mismo color, sus capacidades combinatorias son diferentes, de ahí que puedan resultar anómalas combinaciones del tipo *mesa morena o *chica marrón.

c) Las expresiones institucionalizadas. Son de carácter práctico y sirven para interpretar lo que la otra persona está diciendo. Podemos mencionar: mucho gusto de conocerlo, claro que si, un momento por favor, disculpe la molestia, es un placer.

\section{Trabajo en clase}

La forma de trabajar en clase que adoptamos basándonos en la metodología anteriormente expuesta es variada y centrada en las necesidades de los alumnos. Para ello explicamos el para qué de algunas actividades, los objetivos a alcanzar a corto, mediano y largo plazo y hacemos que los alumnos comprendan que ellos mismos son parte esencial de su proceso de aprendizaje y que deben involucrarse en el, participar. También es importante, dentro de las posibilidades de cada grupo y/o institución, elegir contenidos acordes a los intereses de los alumnos, sus edades y tenerlos en cuenta al momento de planificar las actividades. Las clases planificadas implican conocimiento de 
las metas que queremos lograr y facilitan la distribución de los tiempos, por supuesto sin descartar un margen de flexibilidad que permita adecuar la tarea a situaciones no previstas. Una buena planificación del material es importante para alcanzar las metas en los tiempos propuestos.

Aprender es un proceso dinámico y constante en el que los tiempos no son para todos iguales. Es fundamental que nuestros alumnos entiendan esto para evitar frustraciones en el momento de producir la lengua extranjera y que a la vez comprendan que el error es parte de ese proceso, equivocarse forma parte del aprender y crecer en el conocimiento. Es bueno aprender del error, buscar diferentes alternativas y superarse. Para optimizar el tratamiento del error, lo ideal es corregir en el momento oportuno o preciso en el que el error surge, sin que ello signifique entorpecer la función comunicativa, por ejemplo si corregir implica interrumpir una conversación o una discusión. Puede tratarse el error de forma natural y aprovecharlo para sistematizar, aclarar conceptos y profundizar sobre algún contenido.

Consideramos que es crucial que evaluemos no solo el proceso de aprendizaje (en forma continua) sino también los resultados, ya que cuanto más fructífero sea el primero, más positivos serán los segundos. Un proceso no será del todo valido si no hay resultados. Este concepto de proceso enfocado a resultado implicará a la vez un mayor compromiso de ambas partes.

\section{Propuestas didácticas}

A fin de llevar a cabo actividades que permitan sistematizar el aprendizaje de la lengua extranjera a través del Método Léxico es importante que el material que se vaya a usar para tal fin sea especialmente seleccionado (el uso de material real como artículos de un diario o un video de un comercial de radio o televisión, por ejemplo) para que los alumnos puedan aprender de forma activa. Es fundamental que los alumnos a través de las actividades diseñadas por sus profesores puedan por ejemplo, comprender un mensaje o el significado de un texto, deducir significados por contexto, asociar relacionar imágenes y chunks, definiciones y chunks y chunks entre sí, usar esos chunks y sus combinaciones, expresarse usando el nuevo vocabulario, cuestionar los diferentes usos del vocabulario (Maíz, n.d).

Dentro de las tareas de clase que pueden realizarse para afianzar el aprendizaje desde un enfoque léxico, es decir donde el material no se vea fragmentado, se pueden mencionar las siguientes:

- Dirigir la atención de los alumnos hacia colocaciones léxicas (lexical collocations). Es una buena práctica, sobre todo para que los alumnos se familiaricen con los nuevos conceptos, focalizar el uso en la lengua madre y buscar ejemplos claros que demuestren la idea. 
- Realzar el uso de dichas colocaciones. Al escribir vocabulario nuevo en el pizarrón, por ejemplo, es aconsejable hacerlo en forma de unidades léxicas y no en palabras aisladas.

- Leer o escuchar cada diálogo o conversación o texto al menos dos veces. Repetir ciertas partes, por ejemplo, expresiones idiomáticas.

- Pedir a los alumnos que de a pares o en pequeños grupos identifiquen chunks y los usen en otros contextos.

- Hacer juegos del tipo memo-test usando unidades léxicas recientemente aprendidas.

- Pedir a los alumnos que busquen alguna de las unidades léxicas aprendidas recientemente dentro de otro tipo de texto (canción, periódico, video clip, etc.)

- Dar ejemplos más que explicaciones y a la vez pedir que los alumnos den sus propios ejemplos. Es mucho más fácil recordar aquello que nos es significativo o importante.

- Mostrar cómo se usan las palabras y expresiones. Buscar siempre situaciones que sean de interés para el grupo de alumnos, teniendo en cuenta sus edades gustos, niveles de conocimiento de la lengua en estudio, realidad en la que viven.

- Usar diccionarios (monolingües) y enseñar a usarlos. Los alumnos pueden recurrir a diccionarios en internet.

- Pensar en juegos del tipo pictionary y hacer que los alumnos provean sus propias definiciones también.

- Señalar los grupos de palabras que sugieran situaciones y siempre dar ejemplos contextualizados. Además, es interesante que los alumnos puedan al mismo tiempo elaborar sus ejemplos.

- Comparar situaciones. Contrastar situaciones. Buscar otras situaciones en las cuales se pueda aplicar una misma unidad léxica.

- Usar las nuevas expresiones en contextos familiares para los alumnos y además que sean de su interés dentro de las posibilidades.

- Incentivar a los alumnos a que registren el nuevo léxico en grupos de palabras (frases y oraciones) y no solo palabras sueltas. Una manera de hacerlo es usando frases o colocaciones cuando se escribe algo nuevo en el pizarrón, por ejemplo. Una buena opción para poner en práctica es que cada alumno cree su propio diccionario con ejemplos.

- Tener en la carpeta o cuaderno de clase una sección de vocabulario en la que los alumnos puedan libremente hacer anotaciones según sus preferencias. Por ejemplo, "grupos de palabras que son difíciles de escribir", "grupos de palabras que me gustan", "grupos de palabras que son parecidos", etc. 


\section{Inteligencias Múltiples}

Aprender a través de una variedad de experiencias nos permite entender que nosotros somos capaces de aprender a lo largo de toda nuestra vida y cómo los demás adquieren conocimientos y desarrollan sus habilidades. En nuestro rol docente es fundamental que prestemos atención a las diferencias que existen entre nuestros alumnos y que tratemos de usar ese conocimiento para personalizar la enseñanza y la evaluación. No todos los alumnos son iguales y por ende no todos aprenden de la misma manera.

Tradicionalmente la inteligencia se definía operacionalmente como la habilidad para resolver ítems en tests que medían primordialmente las habilidades lingüísticas y lógico matemáticas de los individuos (Gardner, 1993). Dentro del sistema escolar se valora, en regla general, a aquellos alumnos que tienen un buen vocabulario, capacidad para analizar material de lectura, resolver problemas matemáticos complejos, memorias capaces de retener grandes cantidades de información y rapidez para solucionar problemas. Básicamente quienes tienen capacidades lingüísticas y lógico- matemática desarrolladas, quienes hacen un buen uso de las palabras y los números o bien quienes saben demostrarlo. Según Gardner la inteligencia es mucho más que esto.

Gardner (1983) define a la inteligencia como "el potencial bio-psicológico para procesar información que puede activarse en un contexto cultural para resolver problemas o crear productos de valor dentro de la cultura". Dentro de la definición pueden verse algunos aspectos clave. Gardner habla de un potencial, por tanto está implicando una posibilidad de cambio, de desarrollo, de crecimiento, algo no estático. Otro concepto crucial es de contexto cultural. El ámbito cultural en el cuál el individuo está inmerso le va a dar herramientas que pueden llegar a ser de uso exclusivo de esa cultura o medio. Por ejemplo, un niño que vive en una zona rural probablemente desconozca algunos usos de herramientas de tecnología que un niño de la ciudad usa a diario. Sin embargo, es probable que frente a una tormenta eléctrica sea el niño del campo y no el de la ciudad el que sepa dónde buscar un refugio seguro. Esto no hace que uno sea más inteligente que otro. Son distintas maneras de resolver problemas satisfactoriamente utilizando diferentes potenciales.

Es importante notar que si bien según Gardner (1983) todos los individuos poseen ocho tipos distintos de inteligencia (lingüística, lógico-matemática, musical, espacial, kinestésica, interpersonal, intrapersonal, naturalista) y algunos están más desarrollados que otros según los individuos, todos pueden desarrollarse y fortalecerse. Prestar atención a estas diferencias entre los alumnos y usar ese conocimiento para personalizar la enseñanza y evaluación serán entonces elementos fundamentales en la planificación de las clases.

Algunas estrategias que se pueden usar dentro de la clase de inglés y focalizándonos dentro del marco del Método léxico en particular para fomentar el desarrollo de las distintas inteligencias son: 
- Jugar al scrabble y hacer crucigramas (Inteligencia lingüística). Es importante aquí enfatizar el uso de unidades léxicas, por ejemplo pidiendo a los alumnos que hagan oraciones, o piensen en combinaciones en las que se usen las palabras que aparezcan en el juego.

- Escribir e-mails, mensajes de texto, poemas y diarios personales (Inteligencia lingüística e intrapersonal). Esto puede verse como una actividad real.

- Identificar secuencias de eventos (Inteligencia lingüística). Ideal para ejercitar algunas expresiones como: primero, luego, entonces. Además es una actividad que facilita el proceso de escritura y composición.

- Crear líneas de tiempo (Inteligencia lógico- matemática)

- Comparar textos y dar opiniones sobre los mismos (Inteligencia lógicomatemática, intrapersonal, interpersonal y lingüística). Al hablar de texto no nos referimos exclusivamente a la palabra escrita de forma tradicional en un libro o periódico sino a las variedades de género a las que se enfrentan los alumnos a diario (internet, chats, videoclips, series de televisión, películas)

- Hacer tormentas de ideas- brainstorming- (Inteligencia lógico- matemática, lingüística, kinestésica)

- Analizar errores (Inteligencia lógico- matemática)

- Jugar al pictionary (Inteligencia espacial)

- Ver vídeos, usar mapas y gráficos (Inteligencia espacial)

- Hacer mapas que indiquen cómo llegar a un lugar partiendo desde otro y poder explicarlo (Inteligencia espacial y lingüística). Otra opción es dibujar el mapa escuchando las indicaciones que se dan.

- Tomar notas usando colores (Inteligencia espacial)

- Caminar al leer (Inteligencia kinestésica)

- Hacer role-play (Inteligencia kinestésica, interpersonal)

- Crear efectos sonoros de una historia (Inteligencia musical)

- Escribir (o buscar) una canción que se identifique con algún texto trabajado en clase (Inteligencia musical)

- Encontrar canciones que se refieran a los contenidos de clase (Inteligencia musical, lingüística)

- Trabajar en pares, grupos (Inteligencia interpersonal)

- Debatir, entrevistar, relatar (Inteligencia interpersonal)

- Usar adjetivos para describir gente (Inteligencia interpersonal)

- Usar adjetivos para describirse (Inteligencia intrapersonal)

- Escribir una breve autobiografía (Inteligencia intrapersonal)

- Reflexionar sobre el propio desempeño en la clase (Inteligencia intrapersonal). Es importante permitir espacios para pensar sobre la tarea realizada y 
evaluar las propias fortalezas y debilidades. Es importante crear espacios de silencio y acostumbrar a los alumnos a pensar y poder expresar sus pensamientos.

- Separar lo que se conoce de lo que no se conoce (Inteligencia intrapersonal)

- Describir sueños (Inteligencia intrapersonal)

- Usar material de Greenpeace, Fundación Vida Salvaje (Inteligencia naturalista)

- Ver películas y documentales sobre distintos temas y reflexionar sobre ellos (Inteligencia intrapersonal)

- Asociar letras o palabras a plantas o animales (Inteligencia naturalista)

Es importante notar que dentro del marco teórico expuesto en este trabajo, existe la posibilidad de trabajar interdisciplinariamente. La manera más provechosa de poner en práctica esta teoría es trabajando en proyectos institucionales en los cuales se pueden combinar diferentes áreas curriculares. Por ejemplo, un proyecto sobre especies animales en peligro de extinción puede hacerse desde las matemáticas, calculando los porcentajes de animales en peligro en distintas partes del planeta (inteligencia lógico-matemática), desde el área de música, creando una canción, una historia o un poema que refleje el tema en discusión (inteligencia musical y lingüística), de las ciencias naturales, observando hábitats y clasificándolos (inteligencia naturalista), de las ciencias sociales, a través de encuestas (inteligencia interpersonal y lingüística) y procesando la información (inteligencia lógico-matemática), de las artes, dibujando o buscando trabajos de artistas conocidos sobre el tema en discusión.

\section{Conclusión: Hacia una práctica reflexiva}

En cada aula y en cada clase en particular, ocurren a diario una serie de acontecimientos que el docente puede utilizar para desarrollar la capacidad de entender e interpretar la enseñanza. Muchas veces se desestiman estos eventos y se permite que todos los otros momentos del día tengan prioridad. Sin embargo, algunos momentos y experiencias pueden ser la base de una reflexión crítica de la tarea si los docentes pueden encontrar la forma de capturar los pensamientos y reacciones que surgen de esas oportunidades. Partiendo de esta base, los docentes pueden desarrollar estrategias de cambio o de intervención, según sus necesidades, encontrar maneras de reflexionar y explorar sus experiencias áulicas a través de la auto-observación y autoevaluación. Desarrollar la necesidad de reflexionar y crear el espacio para examinar las propias creencias, actitudes y supuestos sobre la propia práctica así como el usar esa información para la reflexión constituyen estrategias valiosas para el desarrollo profesional.

La tarea de enseñar es un constante desafío, un constante andar por caminos que no siempre son fáciles de transitar y que a menudo nos llevan a 
preguntarnos si vamos en la dirección correcta o hasta si vale la pena recorrerlos. Sin embargo, son muchos los frutos que se pueden recoger de una buena tarea docente. Nuestra tarea, aunque a veces parezca insignificante o sufra en los últimos tiempos de cierto desprestigio público, es simplemente una oportunidad de dar lo mejor de nosotros mismos en cada clase, de demostrar que vale la pena crecer, estudiar y superarse.

\section{Referencias Bibliograficas}

- $\quad$ Armstrong, T. (1993). 7 Kinds of Smart: Identifying and Developing Your Many Intelligences. New York: Plume.

- $\quad$ Arnold, W. \& Westwood, P. (2004). Meeting individual needs with young learners. ELT Journal, 58/4. Oxford University Press.

- Crystal, D. (2005). How language works. How babies babble, words change meaning, and languages live or die. New York: Penguin Books.

- Cook, V. (1997). Second Language Learning and Language Teaching. London: Arnold.

- Diseño Curricular de Lenguas Extranjeras (2001). Gobierno de la Ciudad Autónoma de Buenos Aires. Secretaría de Educación.

- Gardner, Howard. (1983). Frames of Mind: The Theory of Multiple Intelligences. New York: Basic.

- Gardner, Howard. (1993). Multiple Intelligences: The Theory in Practice. New York: Basic.

- Gardner, Howard. (2000). Intelligence Reframed: Multiple Intelligences for the 21st Century. New York: Basic.

- Higueras, M. (2009). Aprender y enseñar léxico. Didáctica del español como lengua extranjera. Expolingua 1996. 9, 111-126. Consultado del sitio web www.marcoele.com/ descargas/expolingua1996-higueras.pdf

- Islam, C. (2003). Lexical Approach 1 - What does the lexical approach look like? TeachingEnglish. British Council. Consultado del sitio web www.teachingenglish.org.uk/articles/ lexical-approach-1-what-does-lexical-aproach-look

- Islam, C. (2003). Lexical Approach 2 - What does the lexical approach look like? TeachingEnglish. British Council. Consultado del sitio web www.teachingenglish.org.uk/articles/ lexical-approach-look

- Gvirtz, S. \& Palamidessi, M. (2008). El ABC de la tarea docente: currículum y enseñanza. Buenos Aires: Aique Grupo editor.

- Larsen-Freeman, D. (2000). Techniques and Principles in Language Teaching. Oxford: Oxford University Press

- Lewis, M. (1998). Implementing the Lexical Approach. Putting Theory into Practice. Hove: Language Teaching Publications.

- Lewis, M. (2002). The Lexical Approach. The State of ELT and a Way Forward. London: Thomsom Heinle.

- Maiz, D. (nd). La enseñanza del léxico en ELE. Aspectos metodológicos y didácticos. Universidad de Rosario. Consultado del sitio www.fhuc.unl.edu.ar/ejes_teamatco 0 espanol_como_lengua_extranjera/maiz.pdf

- Mc Kay, S (s/f). El profesor reflexivo: guía para la investigación del comportamiento en el aula. Portafolio SBS- 2. Buenos Aires: SBS Editora.

- Oxford, Rebecca L. (1990). Language Learning Strategies. What every teacher should know. New York: Newbury House Publishers. 
- $\quad$ Richards, J. \& Lockhart, C. (1996) Reflective Teaching in Second Language Classrooms. Cambridge: Cambridge University Press

- $\quad$ Richards, J. \& Rodgers, T. (2005). Approaches and Methods in Language Teaching. Cambridge: Cambridge University Press.

- $\quad$ Richards, J. (s/f) La enseñanza comunicativa del lenguaje. Portafolio SBS- 53 Buenos Aires: SBS Editora.

- $\quad$ Scrivener, J. (2005). Learning Teaching. Oxford: Macmillan.

- $\quad$ Sutherland, K. (2006) The Theory of Multiple Intelligences: Language teaching applications (publicación electrónica) Consultado del sitio http://www.shareeducation.com.ar/ past $\% 20$ issues2/SHARE\%20168.htm

- Williams, M. \& Burden, R. (2000) Psychology for Language Teachers. A Social Constructivist Approach. Cambridge: Cambridge University Press. 